\title{
Validation of Intramural Wavefront Reconstruction and Estimation of 3D Conduction Velocity
}

\author{
Wilson W Good ${ }^{1}$, Karli K Gillette ${ }^{2}$, Jake A Bergquist ${ }^{1}$, Brian Zenger ${ }^{1}$, Jess Tate $^{1}$, \\ Lindsay C Rupp ${ }^{1}$, Devan Anderson ${ }^{1}$, Gernot Plank ${ }^{2}$, Rob S MacLeod ${ }^{1}$ \\ ${ }^{1}$ Scientific Computing and Imaging Institute, Biomedical Engineering, University of Utah, Salt Lake \\ City, UT, USA \\ ${ }^{2}$ Institute of Biophysics, Medical University of Graz, Graz, Austria
}

\begin{abstract}
Introduction: Changes in conduction velocity are indicative of a wide variety of cardiac abnormalities yet measuring conduction velocity is challenging, especially within the myocardial volume. In this study we investigated a novel technique to reconstruct activation fronts and estimate three-dimensional (3D) conduction velocity $(C V)$ from experimental intramural recordings.

Methods: From the intermittently sampled electrograms we both reconstruct the activation profile and compute the reciprocal of the gradient of activation times and a series of streamlines that allows for the CV estimation.

Results: The reconstructed activation times agreed closely with simulated values, with $50 \%$ to $70 \%$ of the nodes $\leq 1 \mathrm{~ms}$ of absolute error. We found close agreement between the CVs calculated using reconstructed versus simulated activation times. Across the reconstructed stimulation sites we saw that the reconstructed $C V$ was on average $3.8 \%$ different than the ground truth $C V$.

Discussion: This study used simulated datasets to validate our methods for reconstructing $3 D$ activation fronts and estimating conduction velocities. Our results indicate that our method allows accurate reconstructions from sparse measurements, thus allowing us to examine changes in activation induced by experimental interventions such as acute ischemia, ectopic pacing, or drugs.
\end{abstract}

\section{Introduction}

Changes in conduction velocity (CV) within the heart are indicative of a wide variety of cardiac abnormalities and arrthymias, including sudden cardiac death (SCD). The clinical study of CV under conditions like SCD, however, is challenging due to though the short time frame between the onset of symptoms and the cessation of cardiac function. A large range of experimental interventions on large animals in which high density electrical recordings can be made in the intramural space, are therefore essential to improve our understanding of dynamic electrical events that may ultimately result in SCD. Regardless, measurement of $\mathrm{CV}$ is still challenging, and therefore rarely studied in three dimensions throughout the myocardial volume, nor at the level of resolution achieved in this study. [1,2].

In this study, we therefore investigated a novel activation reconstruction approach and a method to estimate threedimensional (3D) CV from intramural needles used experimentally. The technique for estimating $\mathrm{CV}$ throughout the heart volume utilizes the gradient of activation times and a series of streamlines to compute $3 \mathrm{D} \mathrm{CV}$. The approach introduced in this study provide a means to study the formation of these arrhythmias by estimating regional differences in CV throughout a three-dimensional region sampled by intramural needle electrodes.

A key challenge in evaluating the accuracy of activation reconstruction technique, however, is obtaining gold standard data. As there is currently no feasible approach to obtaining the necessary resolution with direct experimental measurement, validation is necessary using bio-physically accurate models of cardiac electrophysiology based from anatomical images of the experimental hearts. For our study, an Eikonal activation sequence with a prescribed orthotropic $\mathrm{CV}$ tensor relating to fiber orientation within CARP was utilized [3,4]. The flexibility of virtual studies allowed us also to generate activation sequences from a range of pacing sites and thus comprehensively evaluate our reconstruction and estimation approaches.

\section{Methods}

\subsection{Wavefront Reconstruction and Esti- mation of 3D Conduction Velocity}

This study used intramural plunge needles, replicating the experimental placement and density, to approximate the conduction velocity (CV) throughout the needle en- 
velope using the reconstructed activation sequences. The activation times recorded on the intramural plunge needles were used to reconstruct the wavefront using a radial basis function (RBF) that interpolated the activation times into the needle envelope. Using the reconstructed activation times the reciprocal of the gradient of activation is estimated and used to compute a series of streamlines. The seed points for around 4000 streamlines were uniformly distributed throughout the needle envelope and were parametrized to follow the activation sequence. The geodesic distance along the streamline was sampled along with the activation time differences at $1 \mathrm{~mm}$ increments to estimate conduction velocity along the streamlines. The values were then smoothed using a simple three element box filter over the length of each streamline. The RBF interpolation, gradient estimation, and streamline generation were all performed using the SCIRun problem solving environment (www.sci.utah.edu/cibc-software/scirun.html). An example of these streamlines, with activation times mapped onto them, can be seen in Figure 2.

\subsection{Experimental Preparation}

The data used for this study was acquired during a series of large animal experiments carried out to study the electrophysiological response to acute myocardial ischemia and a number of other pathologies. $[1,5,6]$ To provide measurements for this study, we conducted a series of experiments using 20-40 transmural plunge needles, each with 10 unipolar electrodes roughly uniformly distributed within the myocardial tissue of the perfusion bed in which ischemia was induced through restriction of coronary flow and elevated heart rates[5]. Figure 1 shows a schematic diagram of a typical intramural needle with 5-10 $\mathrm{mm}$ between each needle. The region sampled by the needles is referred to in this study as the 'needle envelope' and is the enclosed volume created by the spatial distribution of the intramural needles.

\subsection{Computational Model Creation and Simulation}

To generate the validation data, we generated imagebased models of the experimental preparation in similar manner to that outlined by Burton et al.[7]. Meshes were generated using Cleaver with an average edge length of $650 \mu \mathrm{m}$. Rule-based fiber orientations [8] and Universal Ventricular Coordinates (UVCs) [9] were then incorporated, and experimental positions of the needle placements were localized to nodal points on the mesh using meshtool [10]. An Eikonal-based depolarization sequence was computed within CARP from an initial stimulus point defined with UVCs with prescribed CVs of 60,40 , and $20 \mathrm{~cm} / \mathrm{s}$ along the principal, sheer, and normal fiber orientations,

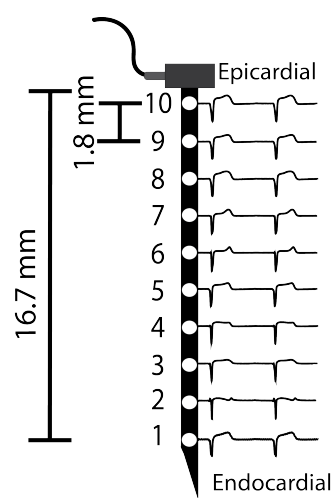

Figure 1. Schematic of an intramural plunge needle used in our experiments. Example signals captured at each depth are located to the right of each electrode. The first electrode is $0.5 \mathrm{~mm}$ below the epicardial surface. Typically 20-30 of these needles are placed into the perfusion bed of the LAD

respectively [3]. The $\mathrm{CV}$ values were chosen to give activation times within the physiological range for canines. To analyze the accuracy of reconstruction into the volume of the needle envelope we stimulated wavefronts from five locations throughout the myocardium. The range of stimulation sites was intended to probe the effect of different wavefront shapes on the reconstruction accuracy throughout the needle hull as well as the estimation of conduction velocity.

From these simulations, we replicated the reconstruction of activation and estimation of $\mathrm{CV}$, comparing the results with the simulated values throughout the volume defined by the needle electrodes. Statistical metrics included the root-mean-square error (RMSE) and the percentage of the hull below $1 \mathrm{~ms}$ of error. Furthermore, the average 3D CV was calculated throughout the reconstructed needle envelope as well as the simulated hull.

\section{Results}

The reconstructed wavefront compared to the calculated activation times had an average RMSE across the five stimulations of $1.58 \mathrm{~ms}$ with $59 \%$ of the needle envelope volume showing an error of less than $1 \mathrm{~ms}$. Figure 3 shows the intramural needle electrodes and the region of the needle envelope that has interpolation error greater than $1 \mathrm{~ms}$. We found the largest error typically occurred at the earliest site of activation if it was not directly sampled by an intramural needle as there is no way to recover earlier than sampled activation times using the RBF interpolation. The majority of the error occurs under the LAD regardless of stimulation site as this is the area in the needle envelope with the least dense sampling. Typically within this sub- 


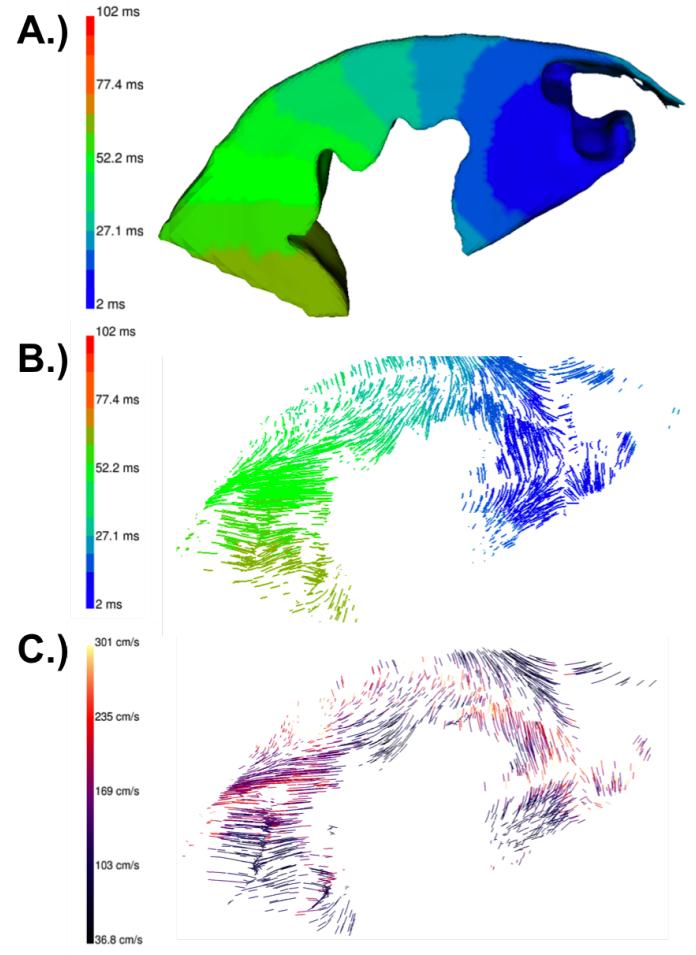

Figure 2. Estimation of 3D CV throughout a portion of the needle envelope. A.) A reconstructed activation sequence through a representative slab of tissue within the needle envelope. B.) A series of generated streamlines parsing the reciprocal of the gradient of activation times with activation times mapped onto the streamlines. C.) Estimated 3D $\mathrm{CV}$ throughout the slab of the needle envelope.

LAD region the error is typically largest near the endocardium.

We analyzed the estimated 3D CVs from both the ground truth and reconstructed activation wavefront to investigate the level of confidence at which $3 \mathrm{D} \mathrm{CV}$ can be estimated with the resolution achieved during our experiments. We found that the CVs that were estimated using the reconstructed activation times agreed with the CVs estimated using the ground truth needle envelope. Furthermore, we saw that the $\mathrm{CV}$ estimated using the ground truth and reconstructed wavefront agreed with the parameterized cellular conduction velocity of the base Eikonal framework.

\section{Discussion}

The purpose of this study was to evaluate the accuracy with which we could reconstruct the activation front in an experimental setting throughout the region measured by the intramural needles. Furthermore, this study will allow us to gain an understanding of the range and variability to
Table 1. RBF-based interpolation error within the needle envelope formed by the intramural needles and mean 3D $\mathrm{CV}$ for the ground truth and reconstructed stimulation site.

\begin{tabular}{ccccc}
\hline \hline $\begin{array}{c}\text { Stim. } \\
\text { Site }\end{array}$ & $\begin{array}{c}\text { RMSE } \\
(\mathrm{ms})\end{array}$ & $\begin{array}{c}\text { Under } \\
\text { Thresh. }\end{array}$ & $\begin{array}{c}\text { Simulated } \\
\text { CV }(\mathrm{cm} / \mathrm{s})\end{array}$ & $\begin{array}{c}\text { Reconstructed } \\
\text { CV }(\mathrm{cm} / \mathrm{s})\end{array}$ \\
\hline A & 2.13 & $49.60 \%$ & 68 & 64 \\
B & 1.37 & $67.97 \%$ & 74 & 76 \\
C & 1.57 & $55.15 \%$ & 74 & 74 \\
D & 1.21 & $67.19 \%$ & 70 & 72 \\
E & 1.63 & $53.55 \%$ & 59 & 55 \\
\hline \hline
\end{tabular}

expect in the computation of 3D CV. This study leveraged the advantages of cardiac computational modeling to validate our ability to reconstruct the activation wavefront using the limited intramural sampling provided by our needle arrays. This study also showed the feasibility of using the volumetric activation front to compute conduction velocity throughout the sampled region.

The ability to accurately reconstruct the activation wavefront and estimate CV has promising appeal for the study of various pathologies that perturb the wavefront. With our results suggesting RMS errors at the level of $2 \mathrm{~ms}$ or less, even subtle changes should be visible with this combination of centimeter scale resolution of the needle electrodes and this novel reconstruction approach. We found that our estimated $\mathrm{CV}$ values were often close to the prescribed values and agreed highly with the ground truth estimations of CV. We have begun to apply this approach to experiments in which we induced acute ischemia, and have found the largest errors to lie under the left anterior descending artery, which is also one of least well sampled regions because of challenges of placing needles close to the artery.

The ability to accurately measure $\mathrm{CV}$ in the myocardium opens many opportunities in the study of arrhythmias, for example, those that arise in the acute phases of ischemia and other cardiac pathologies that one may induce in experiments. A second broad application of these approaches is in the interplay between fiber orientation and propagation in different regions of the heart. Figure 2 shows that even a relatively simple wavefront undergoes a very complex anisotropic activation that introduces a large range in the computed values of CV's. Since the intramural needles allow both pacing and recording, one can envision studies in which pacing from different sites and then measuring (and reconstructing) propagation could lead to as yet unexplored aspects of the spread of excitation.

Limitations in this study have to do primarily with the fact that our validation was based on simulations. There are unavoidable features of the heart used in an experiment that will not be captured faithfully in a geometric model, 


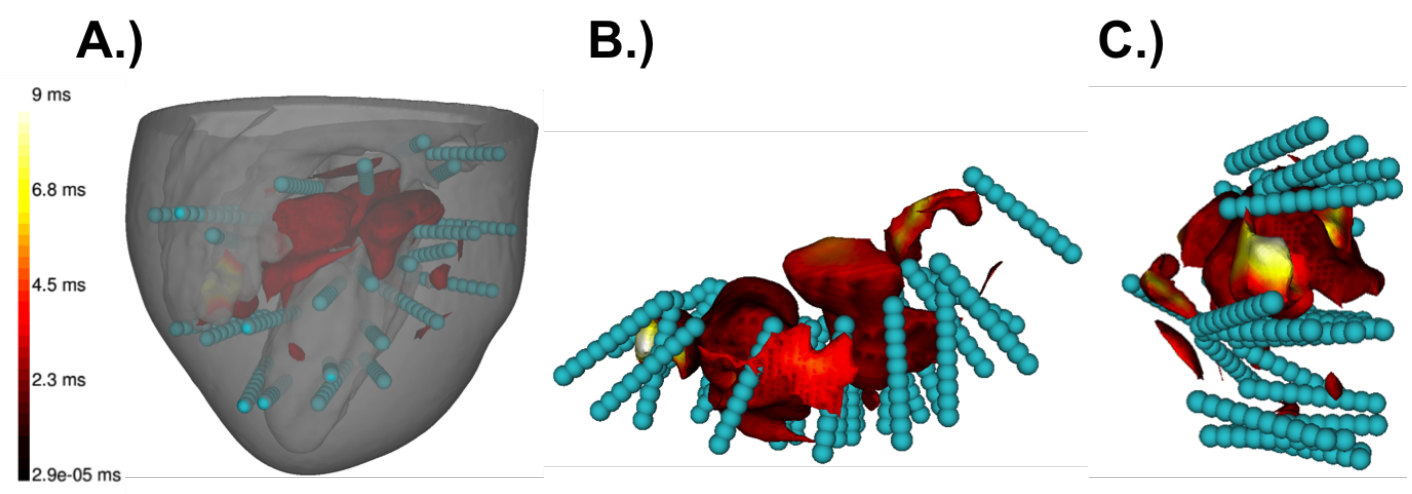

Figure 3. Spatial distribution of low reconstruction accuracy. A.) The experimental heart used in this study showing the intramural electrodes (light blue) and the area of the needle envelope with nodes with absolute error greater than 3 ms. B.) Looking at the needle envelope from a superior aspect showing the volume of relatively low reconstruction accuracy. C.) Looking at the needle envelope from a flanking perspective showing the volume of relatively low reconstruction accuracy.

and even the most sophisticated simulations of propagation have known deviations from reality. In future studies, the volume surrounding the needle electrode locations will be modified in order to better represent concave regions on the endocardium, and capture tissue structure obtained from postmortem MRI scans. We will also review the errors that arise using the Eikonal assumptions of the CARPentry simulations by comparing with full bidomain simulations conducted on the same geometric models.

\section{Acknowledgments}

Support for this research comes from the NIH NIGMS Center for Integrative Biomedical Computing (www.sci.utah.edu/cibc), NIH NIGMS grant no. P41GM103545, the Nora Eccles Treadwell Foundation for Cardiovascular Research.

\section{References}

[1] Good WW, Erem B, Zenger B, Coll-Font J, Brooks DH, MacLeod RS. Temporal performance of laplacian eigenmaps and $3 \mathrm{~d}$ conduction velocity in detecting ischemic stress. Journal of Electrocardiology 2018;

[2] Barnette AR, Bayly PV, Zhang S, Walcott GP, Ideker RE, Smith WM. Estimation of 3-d conduction velocity vector fields from cardiac mapping data. IEEE Transactions on Biomedical Engineering 2000;47(8):1027-1035.

[3] Neic A, Campos FO, Prassl AJ, Niederer SA, Bishop MJ, Vigmond EJ, Plank G. Efficient computation of electrograms and ecgs in human whole heart simulations using a reaction-eikonal model. Journal of Computational Physics 2017;346:191-211.

[4] Vigmond E, Dos Santos RW, Prassl A, Deo M, Plank G. Solvers for the cardiac bidomain equations. Progress in Biophysics and Molecular Biology 2008;96(1-3):3-18.
[5] Aras K, Burton B, Swenson D, MacLeod R. Spatial organization of acute myocardial ischemia. Journal of Electrocardiology 2016;

[6] Zenger B, Good WW, Bergquist J, Tate J, Sharma V, MacLeod RS. Electrocardiographic comparison of dobutamine and bruce cardiac stress testing with high resolution mapping in experimental models ;.

[7] Burton BM, Aras KK, Good WW, Tate JD, Zenger B, MacLeod RS. A framework for image-based modeling of acute myocardial ischemia using intramurally recorded extracellular potentials. Annals of Biomedical Engineering 2018;1-12.

[8] Bayer J, Blake R, Plank G, Trayanova N. A novel rulebased algorithm for assigning myocardial fiber orientation to computational heart models. Annals of Biomedical Engineering 2012;40(10):2243-2254.

[9] Bayer J, Prassl AJ, Pashaei A, Gomez JF, Frontera A, Neic A, Plank G, Vigmond EJ. Universal ventricular coordinates: A generic framework for describing position within the heart and transferring data. Medical Image Analysis 2018;45:83-93.

[10] Neic A, Gsell M, Karabelas E, Prassl A, Plank G. Automating image-based mesh generation and manipulation tasks in cardiac modeling workflows using meshtool. Software X under review 2019;

Address for correspondence:

Name: Wilson William Good

Full postal address: SCI Institute, University of Utah, 72 Central Campus Dr, Salt Lake City, UT 84112

E-mail address: wgood@sci.utah.edu 